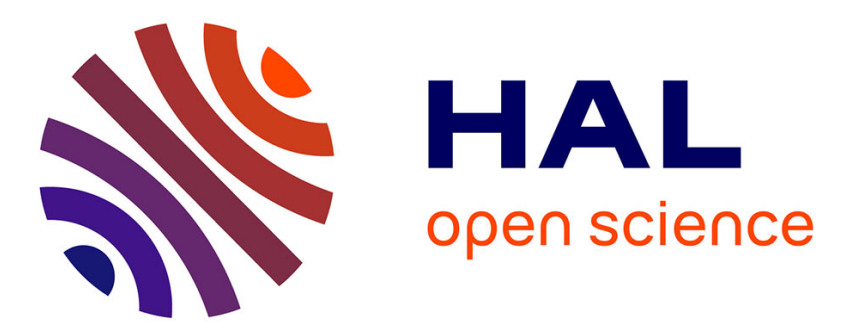

\title{
Evidence of structural reorganization during aggregation of silica nanoparticles
}

\author{
Jules Valente, Frédéric Gruy, Patrice Nortier, Emmanuelle Allain
}

\section{To cite this version:}

Jules Valente, Frédéric Gruy, Patrice Nortier, Emmanuelle Allain. Evidence of structural reorganization during aggregation of silica nanoparticles. Colloids and Surfaces A: Physicochemical and Engineering Aspects, 2015, 468 (5), pp.49-55. 10.1016/j.colsurfa.2014.12.006 . hal-01098376

\section{HAL Id: hal-01098376 https://hal.science/hal-01098376}

Submitted on 29 Jan 2015

HAL is a multi-disciplinary open access archive for the deposit and dissemination of scientific research documents, whether they are published or not. The documents may come from teaching and research institutions in France or abroad, or from public or private research centers.
L'archive ouverte pluridisciplinaire HAL, est destinée au dépôt et à la diffusion de documents scientifiques de niveau recherche, publiés ou non, émanant des établissements d'enseignement et de recherche français ou étrangers, des laboratoires publics ou privés. 


\title{
Evidence of structural reorganization during aggregation of silica nanoparticles
}

\author{
Jules VALENTE, Frédéric GRUY, Patrice NORTIER, Emmanuelle ALLAIN
}

\begin{abstract}
$\underline{\text { Abstract }}$
Silica nanoparticles have been produced by neutralization of sodium silicate. The obtained suspension was unstable because of the high value of the ionic strength of the medium. Aggregation of the particles has been monitored thanks to a combination of in situ turbidity and dynamic light scattering (DLS) measurements. An optical model has been developed to extract both fractal dimension $\mathrm{D}_{\mathrm{f}}$ and primary particle radius $r_{p p}$ of the formed aggregates from these data. The obtained results clearly indicate a densification of the clusters as aggregation proceeds. A correlation between our experimental turbidity values and hydrodynamic radii was found. Comparison with calculated dimensionless numbers showed that a constant Df could not explain the observed trend. A fractal dimension dependent on the number of particles inside the aggregate $\mathrm{N}_{\mathrm{pp}}$ is thus suggested.
\end{abstract}

Keywords: light scattering, aggregates, in situ turbidimetry, restructuring

\section{Introduction}

Colloidal systems are currently used in many fields of application and the potential of such materials is still to be fully explored. As far as silica is concerned, fields of application include controlled abrasion, elastomer reinforcement, whiteness improvement, coating,...[1] Very lately, colloidal suspensions of silica nanoparticles have been highlighted as potential tools for organ repair[2]. When silica is produced from TEOS (tetraethylorthosilicate) using Stöber's method[3], monodisperse isolated particles are obtained. However, when silica is industrially manufactured by neutralization of a silicate solution with acid (precipitated silica) or by hydrolysis of silicon tetrachloride $\mathrm{SiCl}_{4}$ in vapour phase (pyrogenic silica), the resulting powder is made of strong aggregates. These aggregates pile to form large agglomerates. The presence of such structural organization is a prerequisite for certain of the previously mentioned applications. Typically, it has been shown that the hierarchical structures of precipitated silica and carbon black are very similar [4], which explains why the former has progressively replaced the latter as a reinforcing filler in rubbers over the years. However, no consensus exists regarding the morphological properties of the ideal filler [5]. Investigations are thus still conducted to gain control over the morphology of the silica aggregates [6][7][8][9][10]. 
As with many colloids, the aggregates formed by coagulation of silica particles are known to be fractal [11]. This model of aggregate structure [12] is based on the existence of a parameter called the fractal dimension $\mathrm{D}_{\mathrm{f}}$, which varies between 1 and 3 according to the density of the aggregate. Usual values of $\mathrm{D}_{\mathrm{f}}$ are associated with the mechanism of aggregation: 1.8 when aggregation is fast and limited by diffusion of the particles in the suspension and 2.1 when aggregation is slow and limited by the low collision efficiency of the particles. In the case of silica, fractal aggregates can sometimes undergo some restructuring. Aubert and Cannel [13] could form aggregates with fractal dimensions of either 1.75 or 2.05 depending on the conditions of the experiment. The authors also observed that, given enough time, $\mathrm{D}_{\mathrm{f}}$ would naturally increase from 1.75 to 2.05 and that the speed of restructuring was a function of $\mathrm{pH}$.

The origin of such restructuring is still unclear. A possible explanation would be that the aggregated particles are in a shallow secondary potential minimum [14][15][16]. Aggregation would then be reversible: Martin et al. triggered aggregation of silica nanoparticles by addition of salts to their suspension and dispersion of the formed aggregates by dilution[17]. The reversibility of aggregation would allow the primary particles to diffuse inside the aggregates and form denser structures over time. Schlomach and Kind, out of their simulations [18], suggested an Arrhenius-type mechanism for intra-aggregate diffusion. Another theory is based on the nature of the bonds between silica particles: at first, two particles are linked by inter-particle hydroxo bridges between silanols groups [19]. These bonds are easily broken and flexible which gives mobility to the particles inside the aggregate. The hydroxo bridges later evolve into covalent bonds, thus making the aggregation irreversible.

Turbidity is one of the widest-spread techniques to monitor the aggregation of a suspension[20]. It has, unsurprisingly, been applied to study aggregation of silica [21][22]. In this paper, turbidity is used not only to monitor the aggregation of destabilized nanoparticles but also to get structural information about the formed aggregates. An optical model, based on scattering of fractal aggregates, has been built to extract both the fractal dimension and the primary particle size of the aggregates from the experimental turbidity spectra. Evolution of the calculated fractal dimension with time would evidence structural rearrangement of our aggregates. Section 2 of this paper deals with the method of preparation of our silica nanoparticles suspensions and with the characterization techniques employed during this work. The optical model and the associated fitting software we developed are introduced in section 3. In section 4, some of our experimental results are presented and analyzed thanks to our program. Finally, in section 5, the correlation observed between our experimental data is confronted to theoretical considerations.

\section{Material and methods}




\section{Experimental set-up}

Suspensions of silica nanoparticles have been prepared by neutralization of a $\mathrm{SiO} 2$ sodium silicate solution (3.55 $\mathrm{SiO}_{2}: \mathrm{Na}_{2} \mathrm{O}$ ratio) with $8 \mathrm{wt} \%$ sulfuric acid. These solutions have been prepared by dilution of commercially available solutions of $19 \mathrm{wt} \% \mathrm{SiO} 2$ sodium silicate and $72 \mathrm{wt} \%$ sulfuric acid (Chimie Plus) with deionized water. The mixing of reactants has been performed in a $3 \mathrm{~L}$ stirred tank reactor. The temperature has been maintained to $85^{\circ} \mathrm{C}$ during the whole experiment.

The first suspension prepared according to this protocol presented, at the end of the neutralization, a final volume of $1.3 \mathrm{~L}$ and a volume fraction of solid $\Phi_{\mathrm{SiO} 2}$ of $7.5 \times 10^{-3}$. The $\mathrm{pH}$ and the ionic strength in solution were respectively 7.5 and $0.23 \mathrm{M}$ (The ionic strength of the solution has been calculated from the total concentrations of sodium and sulfate ions.). The second one presented a final volume of $1.6 \mathrm{~L}$ and a volume fraction of solid $\Phi_{\mathrm{SiO} 2}$ of $1.22 \times 10^{-2}$. In this case, the $\mathrm{pH}$ and the ionic strength in solution were respectively 8.5 and $0.36 \mathrm{M}$. Once the mixing was over, aggregation of the particles was monitored by a combination of in situ turbidity and DLS measurements until the system gelled. The $\mathrm{pH}$ of the medium did not deviate from its original value during the time of the experiment.

\section{Turbidity measurements}

The turbidity measurements were carried out with a set-up comprising three elements: a light source, a turbidity probe and a commercial spectrometer (Fig. 1). A halogen-deuterium lamp (L7893, Hamamatsu) provides a polychromatic signal in the working range of 350-900nm, which is passed through an optical fibre (600 $\mu \mathrm{m}$ in diameter, T58-457, Edmund Optics) to the probe. The probe has been designed according to Crawley's recommendations [23]: two plano-convex spherical lenses (LPX-5.0-5.2-C, Melles-Griot) insure the parallelism of the light beam across the probe cavity. The length of this cavity sets the optical path length $\mathrm{L}_{\mathrm{opt}}$, which is used in the determination of experimental turbidity. The transmitted signal is passed through a second optical fibre to the spectrometer (USB Red Tide USB650, Ocean Optics). Its wavelength detection range extends from 200 to $1100 \mathrm{~nm}$, with an optical resolution of $2 \mathrm{~nm}$. Turbidity $\tau$ is then calculated according to Eq. 1 . $\mathrm{I}_{0}$ corresponds to the intensity of the incident beam and I to the intensity of the transmitted beam. This calculation is carried out for every available wavelength $\lambda$. The reference spectrum $\mathrm{I}_{0}(\lambda)$ has been recorded before addition of the sulfuric acid to the silicate and thus, before formation of any solid particle.

$\tau=\frac{1}{L_{\mathrm{opt}}} \ln \left(\frac{I_{0}}{I}\right)$

Eq. 1 


\section{Dynamic Light Scattering (DLS) measurements}

Particle size distributions before the gelation point were determined by DLS measurements with a Zetasizer Nano ZS from Malvern Instruments. This appliance is equipped with a He-Ne laser $(\lambda=633$ $\mathrm{nm})$ and a backscatter detector at a fixed angle of $173^{\circ} .5 \mathrm{~mL}$ samples were regularly taken from the suspension. In order to keep them stable until their analysis, each sample was poured in $20 \mathrm{~mL}$ of deionized water at room temperature. Stabilization came from both dilution and cooling of the sample. Besides, $\mathrm{pH}$ of the sample was immediately adjusted to 2 , where aggregation of silica is the slowest according to literature[24], by addition of a small quantity of sulfuric acid.

In this work, only z-average radii are presented. These sizes are the most reliable data given by the Zetasizer Nano ZS as they are directly determined by cumulants analysis of the correlation function. Z-average sizes are therefore intensity averages. Our samples are monomodal with reasonable polydispersity (the polydispersity index PDI determined by cumulants analysis is inferior to 0.3 for all samples) and thus, suited to the use of z-average sizes.

\section{Powder characterization}

After the gelation point, precipitated silica has been collected by centrifugation $(5000 \mathrm{rpm}, 40$ minutes). The obtained paste was redispersed into deionized water and submitted to another 40 minutes of centrifugation in order to purify the sample of residual sodium and sulfate ions. The operation was repeated three times before the silica was dried overnight at $100^{\circ} \mathrm{C}$.

The samples were out-gassed at $160^{\circ} \mathrm{C}$ overnight before $\mathrm{N}_{2}$ physisorption analysis (Micromeritics ASAP 2020). Specific surface areas, determined using the Brunauer-Emmet-Teller (BET) method, were respectively $329.4 \mathrm{~m}^{2} / \mathrm{g}$ and $282.3 \mathrm{~m}^{2} / \mathrm{g}$ for powders obtained after aggregation at $\mathrm{pH} 7.5$ and 8.5 . The equivalent particle diameters are then 8.3 and $9.7 \mathrm{~nm}$. Mesoporosity of the samples was analyzed using the Barrett-Joyner-Halenda (BJH) method. The resulting average pore widths are respectively 8.9 and $10.5 \mathrm{~nm}$.

\section{Optical model}

\section{Scattering theory}

The purpose of working with turbidity at several wavelengths was to be able to extract morphological parameters from the experimental spectra. In the case of silica nanoparticles suspended in water, optical contrast is low: the ratio $m$ of refractive indices for amorphous silica $\left(\mathrm{n}_{\left.\mathrm{SiO}_{2}=1.44\right)}\right)$ and water $\left(\mathrm{n}_{\text {water }}=1.33\right)$ is close to 1 . Particles are small compared to the wavelengths of the incident beam. As a 
result, the Rayleigh-Debye-Gans (RDG) approximation of the Mie theory can be used to calculate the optical properties of the growing aggregates. Turbidity of a suspension of aggregates with $\mathrm{N}_{\mathrm{pp}}$ primary particles of radius $r_{p p}$ is given by Eq. 2. In the frame of this work, the assumption will be made that all the aggregates in the suspension are identical.

$$
\tau=\frac{\phi_{S i O_{2}} * 3}{4 \pi N_{\mathrm{pp}} r_{p p}^{3}} * C_{s c a}
$$

$\mathrm{C}_{\text {sca }}$ is the scattering cross section of an aggregate. In the frame of the RDG approximation, each primary particle inside the aggregate independently scatters the light. In a given direction, the waves scattered by all these particles superpose and generate interferences without multiple scattering. The scattering cross section of the aggregate (averaged over all the possible orientations) is determined by these interferences and obeys Eq. 3.

$$
C_{s c a}=N_{\mathrm{pp}}^{2} \int_{0}^{\pi} S(\theta) * F(\theta) d \theta
$$

$\theta$ is the scattering angle, $\mathrm{F}(\theta)$ is the shape factor, dependent on the primary particle morphology, and $\mathrm{S}(\theta)$ is a structure factor accounting for the spatial arrangement of the particle inside the aggregates. Expressions of both factors are given in Eq. 4 and in Eq. 5. In these equations, $x$ is the product of the wavenumber $k$ and the primary particle radius $\mathrm{r}_{\mathrm{pp}}$, with $k=\left(2 \pi^{*} \mathrm{n}_{\text {water }}\right) / \lambda$.

$F(\theta)=\pi r_{\mathrm{pp}}^{2} * \frac{4}{9}(m-1)^{2} x^{4} *\left(f\left(2 x \sin \left(\frac{\theta}{2}\right)\right)\right)^{2} *\left(1+\cos ^{2} \theta\right) * \sin \theta$

with $f(x)=\frac{3}{x^{3}}(\sin x-x \cos x)$ for spherical primary particles

$$
N_{\mathrm{pp}}^{2} S(\theta)=N_{\mathrm{pp}}+N_{\mathrm{pp}}\left(N_{\mathrm{pp}}-1\right) * \int_{0}^{2} \frac{\sin \left(2 k * r * R_{\mathrm{max}} * \sin \left(\frac{\theta}{2}\right)\right)}{2 k * r * R_{\mathrm{max}} * \sin \left(\frac{\theta}{2}\right)} * D_{p}(r) d r
$$

where $r$ is a dimensionless distance, $\mathrm{R}_{\max }$ is the radius of the circumscribed sphere on the aggregate and $\mathrm{D}_{\mathrm{p}}(\mathrm{r})$ is a pair distribution density corresponding to the arrangement of the primary particles inside the aggregate. The pair distribution density is normalized. Its expression depends on the value of the dimensionless distance $r$. In the case of aggregates with few primary particles, the self-similarity of fractal aggregates is not strictly ensured. Only the relation between the radial number density $\rho$ and the radial coordinate is respected: $\rho(r)=\alpha r^{b}$ with $\alpha$, a proportionality constant, and $b$ equal to $\mathrm{D}_{\mathrm{f}}-3$. Thus, the pair distribution density obeys the following equations: 


$$
D_{p}(r)=\frac{D_{p}{ }^{\prime}(r)}{\int_{0}^{2} D_{p}{ }^{\prime}(r) d r}
$$

- $\mathrm{r}<1$

$$
D_{p}{ }^{\prime}(r)=\int_{1-r}^{1} f_{1}(x) d x+\int_{0}^{1-r} f_{2}(x) d x
$$

- $1 \leq \mathrm{r} \leq 2$

$$
D_{p}{ }^{\prime}(r)=\int_{r-1}^{1} f_{1}(x) d x
$$

With $f_{1}(x)=r * x^{b+1} *\left[1-\left(r^{2}+x^{2}-2 * x r\right)^{1+\frac{b}{2}}\right]$ and

$$
f_{2}(x)=r * x^{b+1} *\left[\left(r^{2}+x^{2}+2 * x r\right)^{1+\frac{b}{2}}-\left(r^{2}+x^{2}-2 * x r\right)^{1+\frac{b}{2}}\right]
$$

\section{Fitting program}

As the aggregates studied in this work are fractal, their radius and number of primary particles are linked by Eq. $6 . \mathrm{R}$ is the radius of the aggregate and $\mathrm{k}_{\mathrm{f}}$ is the fractal prefactor. This factor is still the topic of many investigations and no unique theory exists concerning its value. Some authors express $\mathrm{k}_{\mathrm{f}}$ as an explicit function of $\mathrm{D}_{\mathrm{f}}[25]$ whereas some others give it a specific value depending on the considered aggregation mechanism[26]. Lately, it has even been suggested that $k_{f}$ and $D_{f}$ are not able to completely describe a fractal aggregate and that an extra morphological parameter must be used[27].

$$
N_{\mathrm{pp}}=k_{f}\left(\frac{R}{r_{\mathrm{pp}}}\right)^{D_{f}}
$$

The nature of the radius used in Eq. 6 also has a significant impact on $\mathrm{k}_{\mathrm{f}}$. The result of a DLS analysis is a hydrodynamic radius $R_{\text {hyd }}$. $R_{\text {hyd }}$ corresponds to the radius of the solid sphere with the same diffusion constant as the aggregate. Kaetzel et al. introduced a hydrodynamic dimension of translation $\mathrm{D}_{\mathrm{f}}^{\mathrm{h}, \mathrm{t}}$ and a fractal prefactor of translation $\mathrm{k}_{\mathrm{f}}^{\mathrm{h}, \mathrm{t}}[28]$, which both depend on the fractal dimension $\mathrm{D}_{\mathrm{f}}$. Their dependence on $\mathrm{D}_{\mathrm{f}}$ are given in Eq. 7 and Eq. 8. $\mathrm{D}_{\mathrm{f}}^{\mathrm{h}, \mathrm{t}}$ and $\mathrm{k}_{\mathrm{f}}^{\mathrm{h}, \mathrm{t}}$ are to be used in Eq. 6 when the considered $\mathrm{R}$ is $\mathrm{R}_{\text {hyd. }}$ 
$D_{f}^{h, t}=-4.01+2.10 D_{f}+11.51 D_{f} \exp \left(-1.22 D_{f}\right)$

$k_{f}^{h, t}=5.60-1.41 D_{f}-8.56 D_{f} \exp \left(-1.15 D_{f}\right)$

However, in the calculation of $\mathrm{C}_{\text {sca }}$, the value of $\mathrm{R}_{\max }$ is needed. From the study of Ehrl et al. on simulated fractal aggregates, a relation between the fractal prefactor $k_{f}$ and $D_{f}$ could be inferred, when $R_{\max }$ is the considered radius [29]. Eq. 9 is different from the equation (15) in their paper because the authors used a gyration radius, which is another possible descriptor for a fractal aggregate.

$k_{f}=4.46 * D_{f}^{-2.08} *\left(\frac{D_{f}}{2+D_{f}}\right)^{\frac{D_{f}}{2}}$

A MATLAB program has been designed to deduce both $r_{p p}$ and $D_{f}$ from experimental turbidity spectra. Direct calculation of these parameters from the experimental data would involve the inversion of Eq. 3, which is a complex task. Instead, the program is able to simulate turbidity spectra for given values of $r_{p p}$ and $D_{f}$. When both are fixed, $N_{p p}$ can be inferred from the experimentally determined $R_{\text {hyd }}$ (Eq. 6). Calculation of $\mathrm{C}_{\mathrm{sca}}$ and $\tau$ is then straightforward as $\Phi_{\mathrm{SiO} 2}$ is known. The couple $\left(\mathrm{r}_{\mathrm{pp}}, \mathrm{D}_{\mathrm{f}}\right)$ is numerically adjusted so that the simulated and experimental spectra match at every wavelength. The optimized $r_{p p}$ and $D_{f}$ are the morphological parameters of the aggregate at the time the turbidity spectrum was acquired.

\section{Results}

As similar results were obtained with the two suspensions (see the discussion section), the whole approach is detailed only for the data obtained with aggregation at $\mathrm{pH}$ 7.5.

\section{Monitoring of aggregation}

The time $\mathrm{t}=0 \mathrm{~min}$ corresponds to the end of the mixing of the reactants. Initially, the turbidity value is very low: the silica nanoparticles in the suspension are small and do not scatter much. But over time, the turbidity steadily increases because of the aggregation of the particles (Fig. 2).The turbidity values are higher for shorter wavelengths, which is to be expected in the frame of the RDG approximation. The evolution of turbidity with time is pseudo-linear as was observed by Trompette and Meireles during their study realized with commercial silica suspensions[21]. In the final minutes of the experiment, the linearity is lost. As gelation is close, the optical properties of the suspension change greatly and may not be accurately described by our optical model. As a result, turbidity values after 120 minutes of experiment are not analyzed with our program. 
Aggregation of the particles is also evidenced by the DLS measurements. Initially, the hydrodynamic radius is close to $10 \mathrm{~nm}$. As the radius particle calculated from the specific surface area of the final powder is $4.15 \mathrm{~nm}$, this result would suggest that the particles are already slightly aggregated. The evolution of the hydrodynamic radius with time is also linear. As silica particles are known to generate strong short-range repulsive forces [30][31], a reaction-limited aggregation regime could have been expected. In such case, an exponential evolution of the hydrodynamic radius should theoretically be observed [32]. A decrease in the measured hydrodynamic radius occurred between $t=120$ min and $\mathrm{t}=140 \mathrm{~min}$. The formed aggregates have become too large to be properly characterized by DLS. This confirms that our fitting program should not be used with data acquired after 120 minutes of experiment.

\section{Extraction of morphological parameters}

\section{$\underline{\text { Two fitting parameters }}$}

The fitting program was used to extract morphological parameters from the experimental spectra presented in Fig. 4. Upper and lower limits were set for both $r_{p p}$ and $D_{f}$ : the calculated values of $r_{p p}$ had to be between 3 and $10 \mathrm{~nm}$, calculated values of $D_{\mathrm{f}}$ between 1 and 3.In order to save computational time, the analysis was carried out with spectra consisting of 32 experimental values at selected wavelengths in the range $350-900 \mathrm{~nm}$. The results are presented in Table 1.

The relevance of the returned parameters is questionable. At long times ( $\mathrm{t} \geq 36 \mathrm{~min}), \mathrm{r}_{\mathrm{pp}}$ increases from $3 \mathrm{~nm}$ to $10 \mathrm{~nm}$. As no growth of the particles in the suspension is expected, $\mathrm{r}_{\mathrm{pp}}$ should not vary with time. On the other hand, at short times $(0<\mathrm{t}<36 \mathrm{~min})$, the returned radii are fairly constant and close enough to the $4.15 \mathrm{~nm}$ expected from the BET analysis. Calculated fractal dimensions are rather low compared to the values of the literature. 1.8 is usually the lower limit of experimentally observed fractal dimensions [33]. However, the concept of fractal aggregates has been developed for clusters of several thousands of primary particles. At short times, the characterized aggregates are small and may thus not be described accurately by the fractal theory. The evolution of the calculated $\mathrm{N}_{\mathrm{pp}}$ with time also evidences the lack of physical relevance of these first results. Extracting both $r_{p p}$ and $D_{f}$ from the experimental turbidity spectra appears as a too challenging task for our fitting program.

\section{$\underline{\text { One fitting parameter }}$}

As no growth of the primary particles is expected during the time of the experiment, new series of simulations in which only $D_{f}$ is a fitting parameter and $r_{p p}$ is set to a constant value have been performed. This has been done for $\mathrm{r}_{\mathrm{pp}}=4.2 \mathrm{~nm}$, the value derived from the BET analysis, and for 
$r_{p p}=5.0 \mathrm{~nm}$, which is the average of the fitted radii between $t=5 \mathrm{~min}$ and $\mathrm{t}=22 \mathrm{~min}$. The results of these simulations are reported in Table 2.

The results obtained with both values of $r_{p p}$ follow the same trend: $D_{f}$ is fairly constant from $t=5$ min to $t=22$ min and steadily increases at longer times. $D_{f}$ is lower at short times for the greater $r_{p p}$ value but its final value is rather independent from the chosen $r_{p p}$. In both cases, $D_{f}$ is around 2.2 which is the theoretical fractal dimension for reaction-limited aggregation. The evolution of $\mathrm{D}_{\mathrm{f}}$ with time evidences a restructuring of the aggregates. Besides, evolution of the calculated $\mathrm{N}_{\mathrm{pp}}$ with time is well approximated by an exponential function, which is compatible with reaction-limited aggregation (Fig. 5). The values of $N_{p p}$ are systematically higher for the smaller $r_{p p}$.

\section{Discussion}

Turbidity and hydrodynamic radius were both found to follow a linear evolution over the aggregation time. Despite significant differences in the conditions of operation, a similar behavior was observed at pH 8.5 (Fig. 6). This suggests a certain universality of the restructuring evidenced thanks to our fitting program. However, direct comparison of the two experiments is difficult as the kinetics of aggregation is different in each case.

In order to mitigate these differences and make comparison easier, we calculated dimensionless turbidities $\tau_{\text {adim }}$ : the experimental value was divided by the turbidity the suspension would have if all the primary particles were isolated, ie $\mathrm{N}_{\mathrm{pp}}=1$ (Eq. 10). The resulting value is thus only dependent on the spatial arrangement of the primary particles inside the aggregate in the RDG framework. In the same fashion, the experimental hydrodynamic radius was divided by the radius of the primary particle, which was fixed at $5 \mathrm{~nm}$. According to Eq. 6, this dimensionless radius $\mathrm{R}_{\mathrm{adim}}$ is a function of $\mathrm{N}_{\mathrm{pp}}$ and $D_{f}$ only. The resulting plot is presented in Fig. 7. A linear relation between the two kinds of dimensionless numbers is evidenced, with nearly identical slopes for both sets of data.

$$
\tau_{\text {adim }}=\frac{\tau_{\mathrm{XP}}}{\frac{\phi_{s i O_{2}} * 3}{4 \pi r_{p p}^{3}} * C_{s c a}\left(N_{p p}=1\right)}=\frac{C_{s c a}\left(N_{p p}\right)}{N_{p p} * C_{s c a}\left(N_{p p}=1\right)}
$$

In order to check whether such correlation is specific to our system or can be found in every suspension of aggregates with fractal properties, we calculated theoretical $\tau_{\text {adim }}$ and $\mathrm{R}_{\text {adim }}$ at different values of $D_{f}$ for $N_{p p}$ varying between 1 and 500. For these calculations, $r_{p p}$ was once again fixed at 5 $\mathrm{nm}$. The results are plotted on Fig. 8. For constant $\mathrm{D}_{\mathrm{f}}$, the evolution of $\tau_{\text {adim }}$ significantly differs from what could be observed with our experimental data. In particular, calculated $\tau_{\text {adim }}$ systematically levels 
off for large $\mathrm{R}_{\mathrm{adim}}$ values with the plateau being reached sooner for smaller $\mathrm{D}_{\mathrm{f}}$. However, when using a fractal dimension dependent on $\mathrm{N}_{\mathrm{pp}}$, the results significantly improved. A good linearity was found up to $R_{\text {adim }}=12$. Simulated and experimental values do not superimpose perfectly but the evolutions are quite similar.

$$
D_{f}=1.6+0.6 * \tanh \left(0.003 * N_{p p}\right)
$$

The relation used (Eq. 11) is probably not the best that could be found but it reproduces reasonably well the evolution of fitted $D_{f}$ over time obtained for $r_{p p}=4.2 \mathrm{~nm}$, using the calculated $\mathrm{N}_{\mathrm{pp}}$ in Table 2 (Fig. 9). The idea of a fractal dimension increasing with the number of primary particles inside the aggregate is in agreement with the theoretical considerations proposed by Gmachowski [34]. Besides, it enables us to prove our point: during our experiments, the aggregates underwent some restructuring, leading to larger $\mathrm{D}_{\mathrm{f}}$ over time.

\section{$\underline{\text { Conclusion }}$}

In this paper, we have presented an optical model for scattering of silica aggregates with fractal properties. This model was implemented in a fitting program designed to extract the radius of primary particle $r_{p p}$ and the fractal dimension of aggregate $D_{f}$ from experimental turbidity spectra. Aggregation of two suspensions prepared by neutralization of sodium silicate with sulfuric acid was monitored by DLS and in situ turbidity measurements. The data issued from one of these experiments evidenced a densification of the aggregates with time. A correlation between our experimental turbidity values and hydrodynamic radii was found. By confrontation of these data with theoretical calculations, densification of the aggregates was once again evidenced. A fractal dimension dependent on the number of primary particles $\mathrm{N}_{\mathrm{pp}}$ has been suggested. There is room for improvement of the proposed expression. The kinetics of restructuring may possibly be determined and correlated with the physicochemical parameters of the suspension.

In situ SAXS measurements would also be a valuable asset, as it would enable us to confirm the evolution of $\mathrm{D}_{\mathrm{f}}$ we observed. The small number of primary particles in the aggregates of our suspensions may cause difficulties in the interpretation of SAXS experimental data. The classical method which relies on the use of the function $\mathrm{q}^{-\mathrm{Df}}$ (where $\mathrm{q}$ is the modulus of the scattering vector) may indeed not be applicable in our case. Cryo-TEM observations coupled with image analysis are also considered to validate our results. 
Besides, the analytical approach described in this work could be employed with other systems for which the RDG approximation remains valid. It is typically the case of suspensions of submicronic polymer particles. These systems, as silica, have been widely used in investigations on aggregation. Our method could thus prove to be a valuable tool to obtain time-resolved information about aggregating systems and potentially lead to a better understanding of aggregation in general. 


\section{$\underline{\text { Abbreviations }}$}

$\mathrm{C}_{\mathrm{sca}}=$ scattering cross section $\left(\mathrm{m}^{2}\right)$

$D_{\mathrm{f}}=$ fractal dimension

$\mathrm{D}_{\mathrm{f}}^{\mathrm{h}, \mathrm{t}}=$ hydrodynamic dimension of translation

$\mathrm{D}_{\mathrm{p}}(\mathrm{r})=$ pair distribution density

$\mathrm{F}(\theta)=$ shape factor

$I(\lambda)=$ intensity of the transmitted beam

$\mathrm{I}_{0}(\lambda)=$ intensity of the incident beam

$\mathrm{L}_{\text {opt }}=$ optical path length

$\mathrm{N}_{\mathrm{pp}}=$ number of primary particles in an aggregate

$\mathrm{R}_{\mathrm{adim}}=$ dimensionless radius

$\mathrm{R}_{\text {hyd }}=$ hydrodynamic radius of aggregate $(\mathrm{nm})$

$\mathrm{R}_{\max }=$ radius of the circumscribed sphere on the aggregate $(\mathrm{nm})$

$\mathrm{S}(\theta)=$ structure factor

$\mathrm{BET}=$ Brunauer-Emmet-Teller (method used to determine the specific surface area of a powder for $\mathrm{N}_{2}$ physisorption data)

$\mathrm{BJH}=$ Barrett-Joyner-Halenda (method used to determine the distribution of mesopore sizes of a powder for $\mathrm{N}_{2}$ physisorption data)

DLS $=$ Dynamic Light Scattering

RDG $=$ Rayleigh-Debye-Gans (approximation of the exact Mie scattering theory)

$b=\mathrm{D}_{\mathrm{f}}-3$

$k=$ wavenumber

$\mathrm{k}_{\mathrm{f}=\text { fractal prefactor }}$

$\mathrm{k}_{\mathrm{f}}^{\mathrm{h}, \mathrm{t}}=$ fractal prefactor of translation

$m=$ relative refractive index

$\mathrm{n}_{\mathrm{SiO} 2}=$ refractive index of amorphous silica (1.44)

$\mathrm{n}_{\text {water }}=$ refractive index of water (1.33)

$r=$ dimensionless distance

$\mathrm{r}_{\mathrm{pp}}=$ radius of primary particle $(\mathrm{nm})$

$\lambda=$ wavelength $(\mathrm{nm})$

$\rho(r)=$ radial number density 
$\tau=$ turbidity $\left(\mathrm{cm}^{-1}\right)$

$\tau_{\text {adim }}=$ dimensionless turbidity

$\theta=$ scattering angle $\left(^{\circ}\right)$

$\Phi_{\mathrm{SiO} 2}=$ volume fraction of solid 


\section{$\underline{\text { References }}$}

1. Colloidal silica : fundamentals and applications (2006). Bergna HE, Roberts WO, editors Boca Raton, Fla.; London; New York: Taylor and Francis. 912 p. Accessed 19 June 2012.

2. Meddahi-Pellé, A., Legrand, A., Marcellan, A., Louedec, L., Letourneur, D., and Leibler, L. (2014) Organ Repair, Hemostasis, and In Vivo Bonding of Medical Devices by Aqueous Solutions of Nanoparticles. Angew. Chem., 126,6487-6491. doi:10.1002/ange.201401043.

3. Stöber, W., Fink, A., and Bohn, E. (1968) Controlled Growth of Monodisperse Silica Spheres in Micron Size Range. J. Colloid Interface Sci., 26,62 - \&. doi:10.10160021-9797(68)90272-5.

4. Schaefer, D., Rieker, T., Agamalian, M., et al. (2000) Multilevel structure of reinforcing silica and carbon. J. Appl. Crystallogr., 33,587-591. doi:10.1107S0021889800001199.

5. Schaefer, D., Kohls, D., and Feinblum, E. (2012) Morphology of Highly Dispersing Precipitated Silica: Impact of Drying and Sonication. J. Inorg. Organomet. Polym. Mater., 22,617-623. doi:10.1007/s10904-011-9643-y.

6. Schilde, C., Hanisch, C., Naumann, D., Beierle, T., and Kwade, A. (2013) A novel way to vary the structure of precipitated silica and calcium carbonate aggregates in a wide range by using grinding media during the precipitation process. Chem. Eng. Sci., 94,127-137. doi:10.1016/j.ces.2013.02.042.

7. Knoblich, B. and Gerber, T. (2001) Aggregation in $\mathrm{SiO} 2$ sols from sodium silicate solutions. $J$. Non-Cryst. Solids, 283,109-113. doi:10.1016/S0022-3093(01)00356-8.

8. Wijnen, P., Beelen, T., Rummens, C., and Van Santen, R. (1991) Diffusion-Limited and ReactionLimited Aggregation of Aqueous Silicate Solutions. J. Non-Cryst. Solids, 136,119-125. doi:10.10160022-3093(91)90127-R.

9. Schlomach, J. and Kind, M. (2004) Investigations on the semi-batch precipitation of silica. $J$. Colloid Interface Sci., 277,316-326. doi:10.1016j.jcis.2004.04.051.

10. Baldyga, J., Jasińska, M., Jodko, K., and Petelski, P. (n.d.) Precipitation of amorphous colloidal silica from aqueous solutions-Aggregation problem. Chem. Eng. Sci.,. Accessed 1 June 2012.

11. Lin, M.Y., Lindsay, H.M., Weitz, D.A., Ball, R.C., Klein, R., and Meakin, P. (1989) Universality in colloid aggregation. Nature, 339,360-362. doi:10.1038/339360a0.

12. Meakin, P. (1987) Fractal aggregates. Adv. Colloid Interface Sci., 28,249-331. doi:10.1016/00018686(87)80016-7.

13. Aubert, C. and Cannell, D. (1986) Restructuring of Colloidal Silica Aggregates. Phys. Rev. Lett., 56,738-741. doi:10.1103PhysRevLett.56.738.

14. Chinchalikar, A.J., Aswal, V.K., Kohlbrecher, J., and Wagh, A.G. (2012) Evolution of structure and interaction during aggregation of silica nanoparticles in aqueous electrolyte solution. Chem. Phys. Lett., 542,74-80. doi:10.1016/j.cplett.2012.05.065.

15. Skvarla, J. (2013) Quantitative Interpretation of Anomalous Coagulation Behavior of Colloidal Silica Using a Swellable Polyelectrolyte Gel Model of Electrical Double Layer. Langmuir, 29,8809-8824. doi:10.1021/la401502f. 
16. Johnsson, A.-C.J.H., Camerani, M.C., and Abbas, Z. (2011) Combined Electrospray-Scanning Mobility Particle Sizer (ES-SMPS) and Time-Resolved Synchrotron Radiation-Small-Angle Xray Scattering (SR-SAXS) Investigation of Colloidal Silica Aggregation. Part II. Influence of Aggregation Initiator on Gel Stability. J. Phys. Chem. B, 115,9547-9555. doi:10.1021jp2032753.

17. Martin, J., Wilcoxon, J., Schaefer, D., and Odinek, J. (1990) Fast Aggregation of Colloidal Silica. Phys. Rev. A, 41,4379-4391. doi:10.1103PhysRevA.41.4379.

18. Schlomach, J. and Kind, M. (2007) Theoretical study of the reorganization of fractal aggregates by diffusion. Part. Sci. Technol., 25,519-533. doi:10.108002726350701490912.

19. Depasse, J. (1997) Coagulation of colloidal silica by alkaline cations Surface dehydration or interparticle bridging. J. Colloid Interface Sci., 194,260-262. doi:10.1006jcis.1997.5120.

20. Gregory, J. (2009) Monitoring particle aggregation processes. Adv. Colloid Interface Sci., 14748,109-123. doi:10.1016j.cis.2008.09.003.

21. Trompette, J. and Meireles, M. (2003) Ion-specific effect on the gelation kinetics of concentrated colloidal silica suspensions. J. Colloid Interface Sci., 263,522-527. doi:10.1016S00219797(03)00397-7.

22. Gruy, F. (2001) Formation of small silica aggregates by turbulent aggregation. J. Colloid Interface Sci., 237,28-39. doi:10.1006jcis.2001.7432.

23. Crawley, G., Cournil, M., and Di Benedetto, D. (1997) Size analysis of fine particle suspensions by spectral turbidimetry: potential and limits. Powder Technol., 91,197-208. doi:10.1016/S00325910(96)03252-4.

24. Iler, R.-K. (1979) The Chemistry of silica solubility, polymerization, colloid and surface properties, and biochemistry. New York: J. Wiley \& Sons.

25. Gmachowski, L. (2002) Calculation of the fractal dimension of aggregates. Colloids Surf. Physicochem. Eng. Asp., 211,197-203. doi:10.1016S0927-7757(02)00278-9.

26. Brasil, A.., Farias, T.., Carvalho, M.., and Koylu, U.. (2001) Numerical characterization of the morphology of aggregated particles. J. Aerosol Sci., 32,489-508. doi:10.1016/S00218502(00)00097-5.

27. Heinson, W.R., Sorensen, C.M., and Chakrabarti, A. (2012) A three parameter description of the structure of diffusion limited cluster fractal aggregates. J. Colloid Interface Sci., 375,65-69. doi:10.1016j.jcis.2012.01.062.

28. Kaetzel, U., Bedrich, R., Stintz, M., Ketzmerick, R., Gottschalk-Gaudig, T., and Barthel, H. (2008) Dynamic light scattering for the characterization of polydisperse fractal systems: I. Simulation of the diffusional Behavior. Part. Part. Syst. Charact., 25,9-18. doi:10.1002/ppsc.200700004.

29. Ehrl, L., Soos, M., and Lattuada, M. (2009) Generation and Geometrical Analysis of Dense Clusters with Variable Fractal Dimension. J. Phys. Chem. B, 113,10587-10599. doi:10.1021/jp903557m.

30. Guleryuz, H., Røyset, A., Kaus, I., Filiàtre, C., and Einarsrud, M.-A. (2012) AFM measurements of forces between silica surfaces. J. Sol-Gel Sci. Technol., 62,460-469. doi:10.1007/s10971-0122750-6. 
31. Kobayashi, M., Juillerat, F., Galletto, P., Bowen, P., and Borkovec, M. (2005) Aggregation and charging of colloidal silica particles Effect of particle size. Langmuir, 21,5761-5769. doi:10.1021la046829z.

32. Runkana, V., Somasundaran, P., and Kapur, P.C. (2005) Reaction-limited aggregation in presence of short-range structural forces. Aiche J., 51,1233-1245. doi:10.1002/aic.10375.

33. Wu, H., Lattuada, M., and Morbidelli, M. (2013) Dependence of fractal dimension of DLCA clusters on size of primary particles. Adv. Colloid Interface Sci., 195-196,41-49. doi:10.1016/j.cis.2013.04.001.

34. Gmachowski, L. (2009) Aggregate restructuring by internal aggregation. Colloids Surf. Physicochem. Eng. Asp., 352,70-73. doi:10.1016j.colsurfa.2009.09.052. 


\section{List of figures}

Fig. 1: Experimental set-up for turbidity measurements

Fig. 2: Evolution of experimental turbidity at several wavelenghts with time (the black dashed line is just a guide for the eye to appreciate the linearity of the evolution) $-\mathrm{pH} 7.5$

Fig. 3: Evolution of hydrodynamic radius with time $-\mathrm{pH} 7.5$

Fig. 4: Experimental turbidity spectra used in the fitting program $-\mathrm{pH} 7.5$

Fig. 5: Evolution of the calculated $\mathrm{N}_{\mathrm{pp}}$ with time $-\mathrm{pH} 7.5$

Fig. 6: Comparison of the evolution of turbidity and hydrodynamic radius with time in the two experiments

Fig. 7: Dimensionless turbidity as a function of dimensionless radius - Experimental data

Fig. 8: Dimensionless turbidity as a function of dimensionless radius - Simulations (the black dotted line is just a guide for the eye to appreciate the linearity of the evolution)

Fig. 9: Comparison of the fitted $D_{f}$ with the values issued from $D_{f}\left(N_{p p}\right)=1.6+0.6 * \tanh \left(0.003 * N_{p p}\right)$ 


\section{List of tables}

Table 1: Aggregate morphological parameters extracted from the experimental turbidity spectra at different times of the experiment (Upper and lower limits are respectively $3 \mathrm{~nm}$ and $10 \mathrm{~nm}$ for $\mathrm{r}_{\mathrm{pp}}, 1$ and 3 for $\mathrm{D}_{\mathrm{f}}$ ) $-\mathrm{pH} 7.5$

Table 2: Aggregate morphological parameters extracted from the experimental turbidity spectra at different times of the experiment when $r_{p p}$ is fixed and $D_{f}$ is a fitting parameter (Upper and lower limits are 1 and 3 for $D_{f}$. The calculations are performed successively for $r_{p p}=4.2 \mathrm{~nm}$ and $r_{p p}=5.0 \mathrm{~nm}$ ) $-\mathrm{pH} 7.5$ 


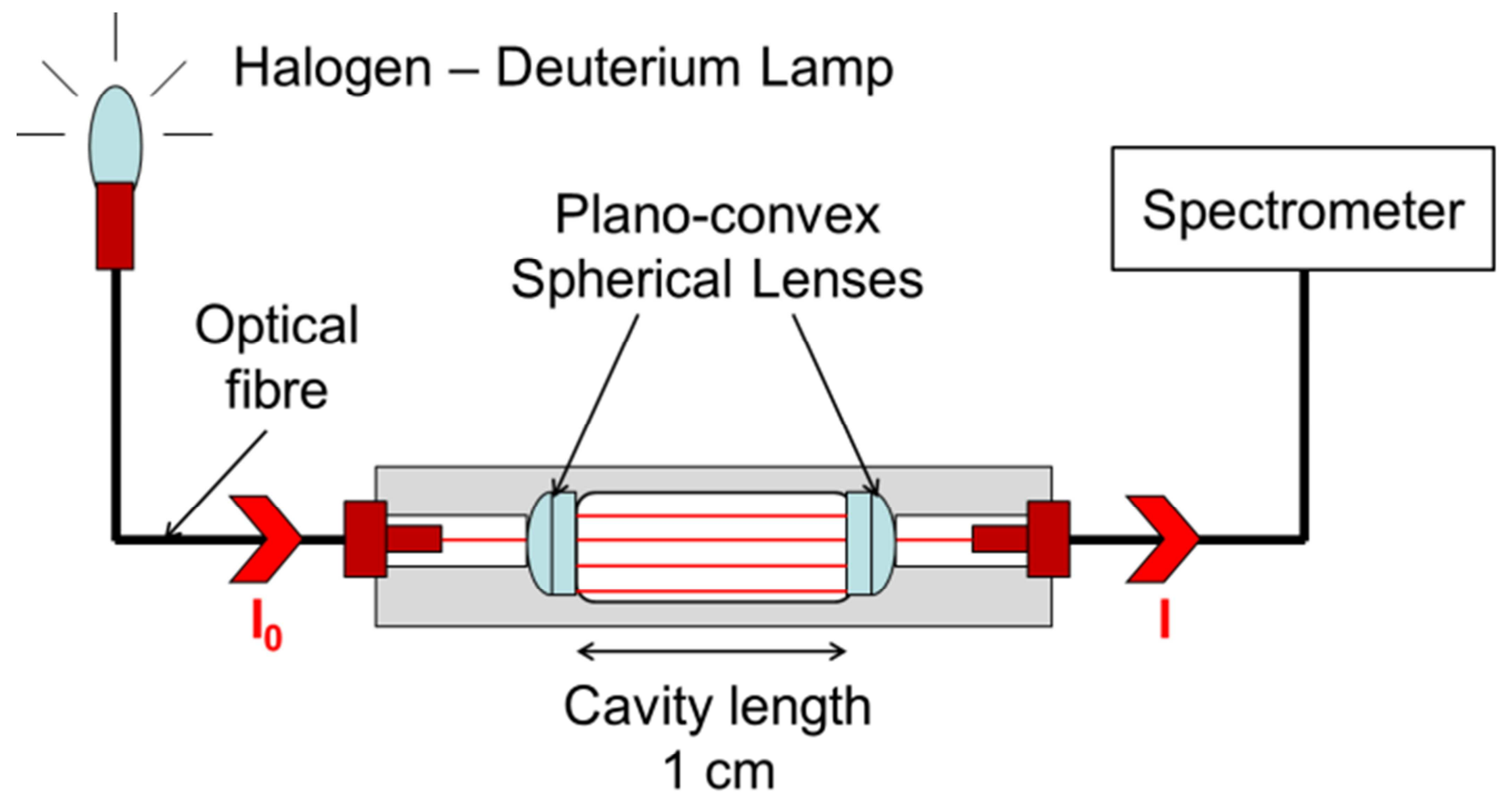

Figure 1: Experimental set-up for turbidity measurements 


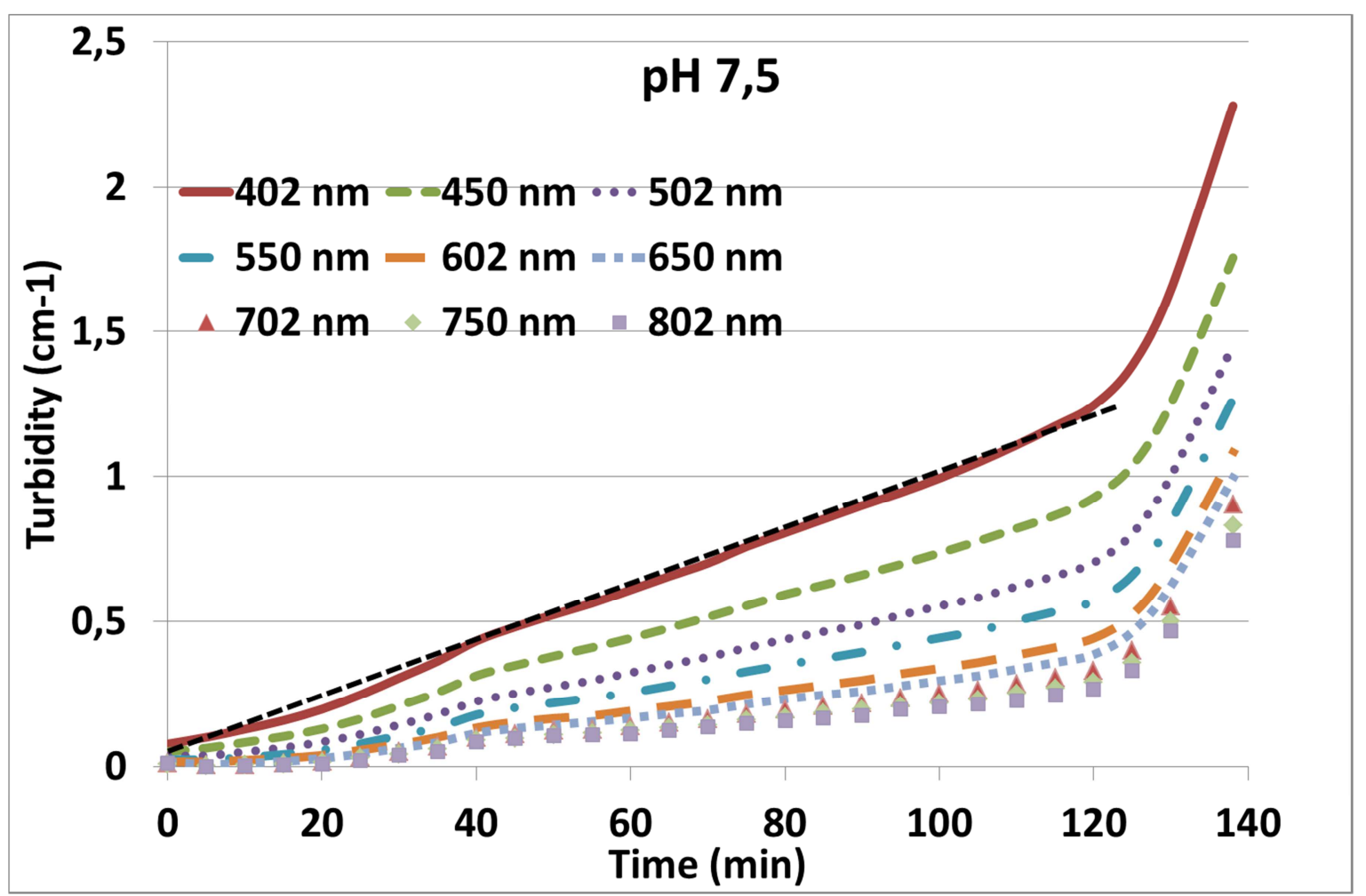

Figure 2: Evolution of experimental turbidity at several wavelenghts with time (the black dashed line is just a guide for the eye to appreciate the linearity of the evolution) - pH 7.5 


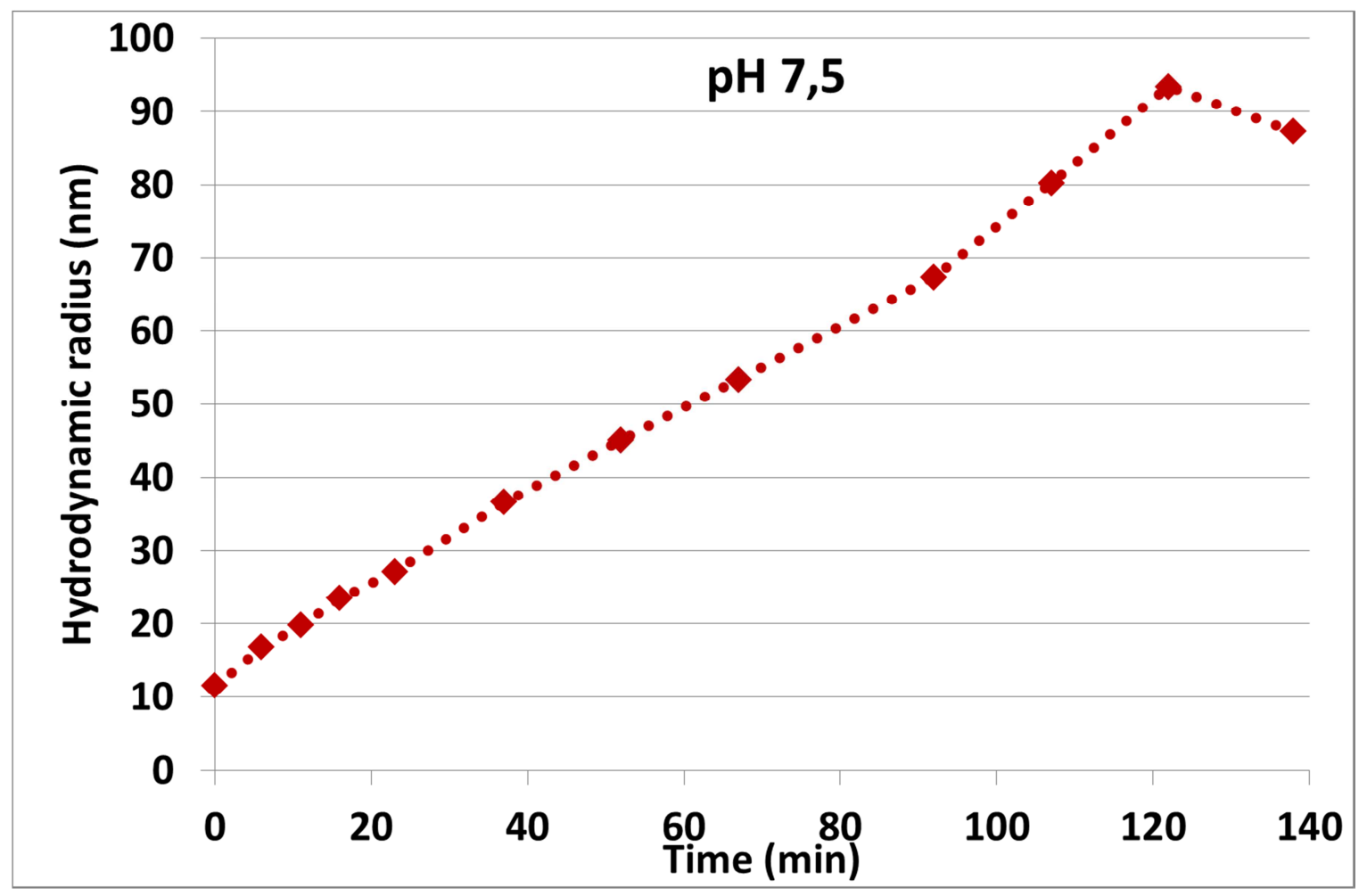

Figure 3: Evolution of hydrodynamic radius with time - $\mathrm{pH}$ 7.5 


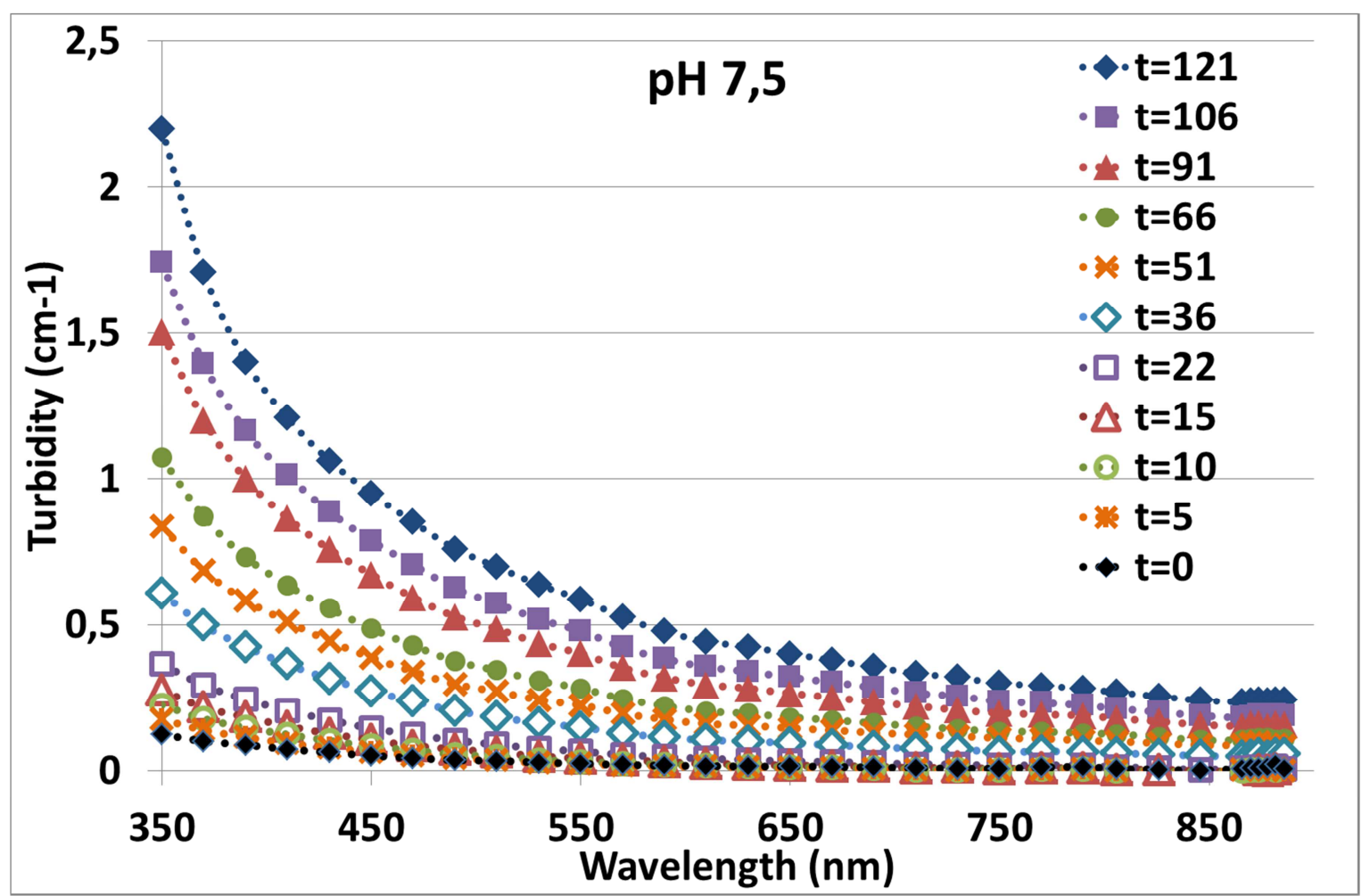

Figure 4: Experimental turbidity spectra used in the fitting program - pH 7.5 


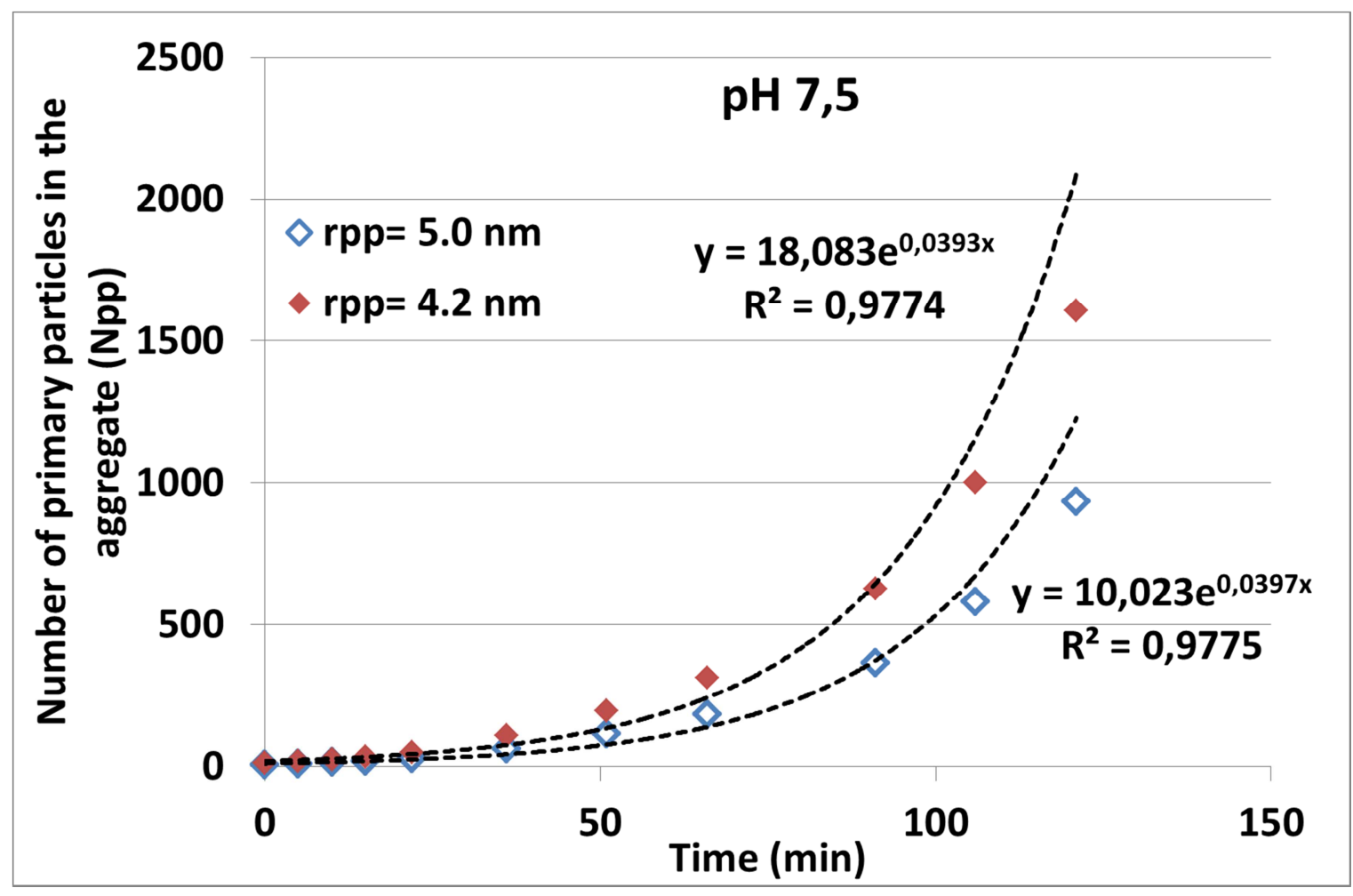

Figure 5: Evolution of the calculated Npp with time - pH 7.5 


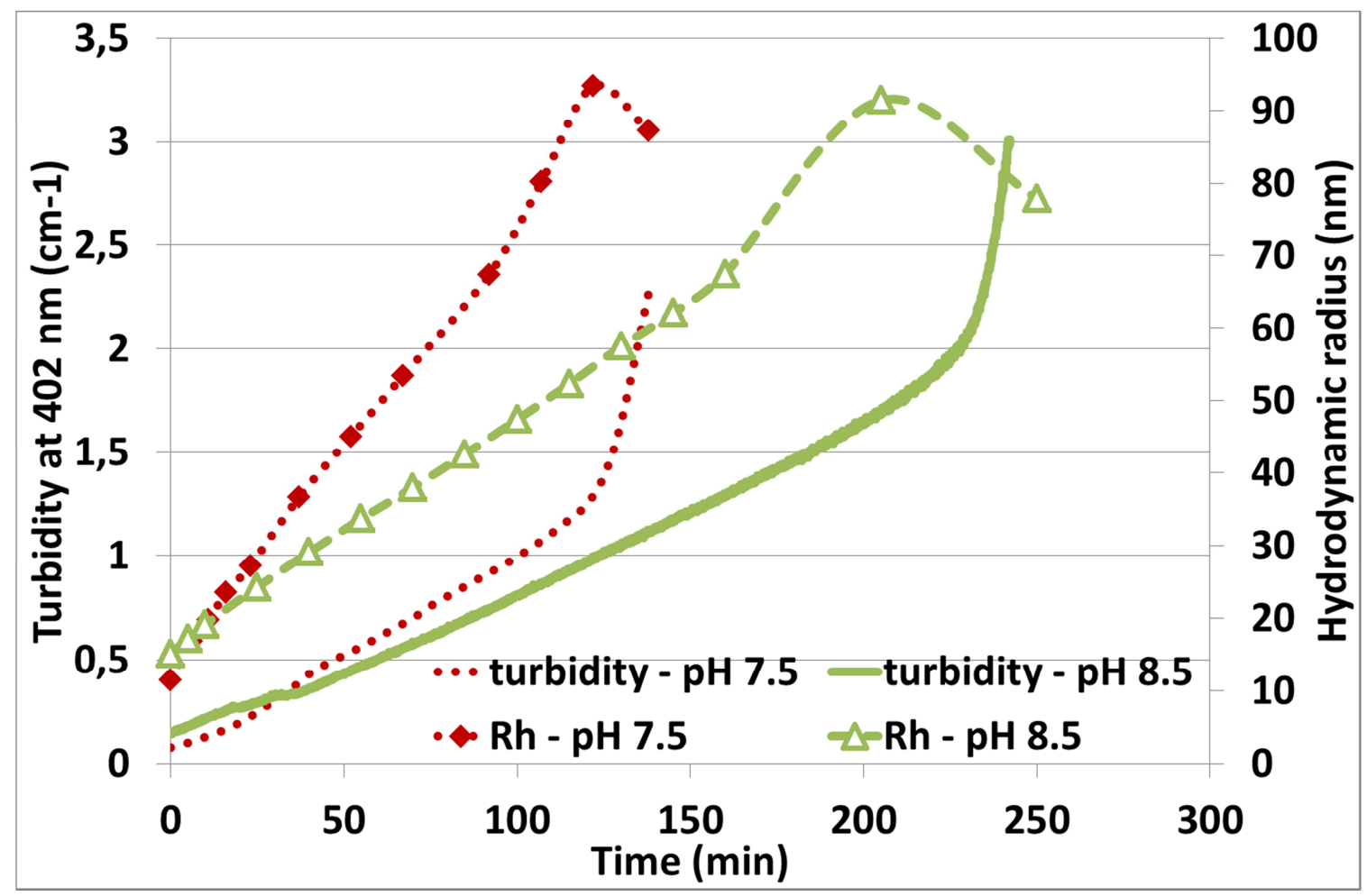

Figure 6: Comparison of the evolution of turbidity and hydrodynamic radius with time in the two experiments 


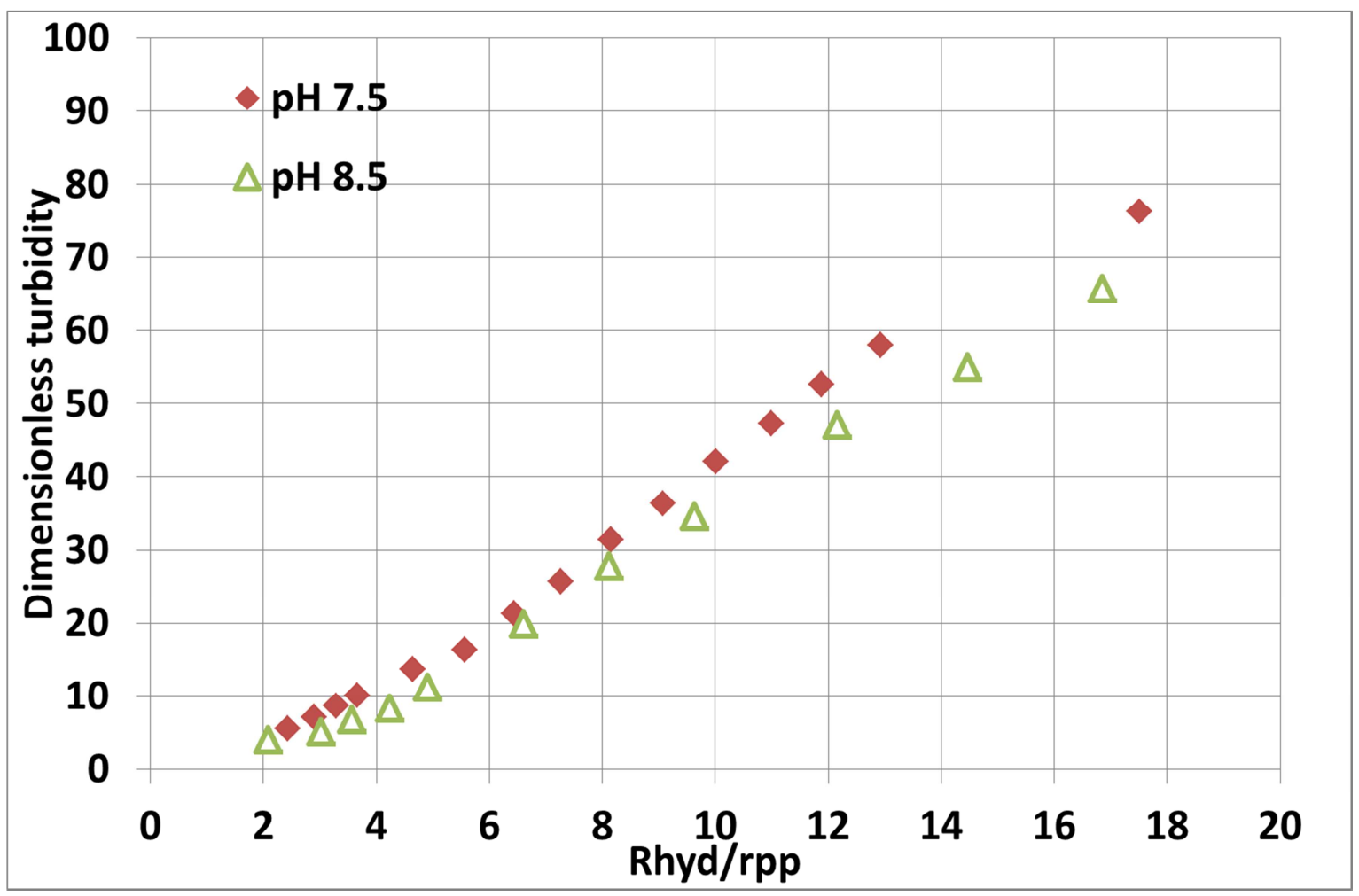

Figure 7: Dimensionless turbidity as a function of dimensionless radius - Experimental data 


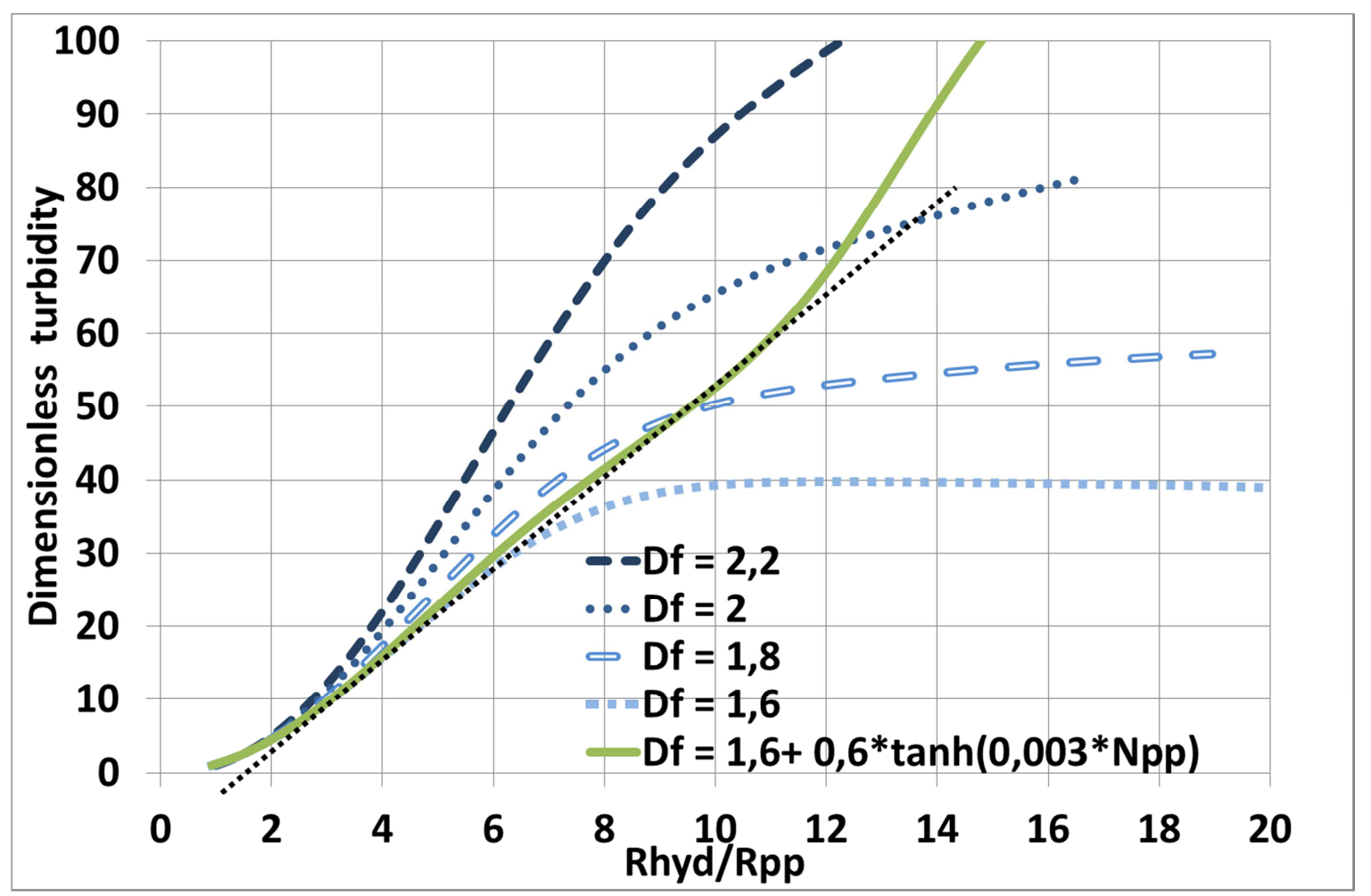

Figure 8: Dimensionless turbidity as a function of dimensionless radius - Simulations (the black dotted line is just a guide for the eye to appreciate the linearity of the evolution) 


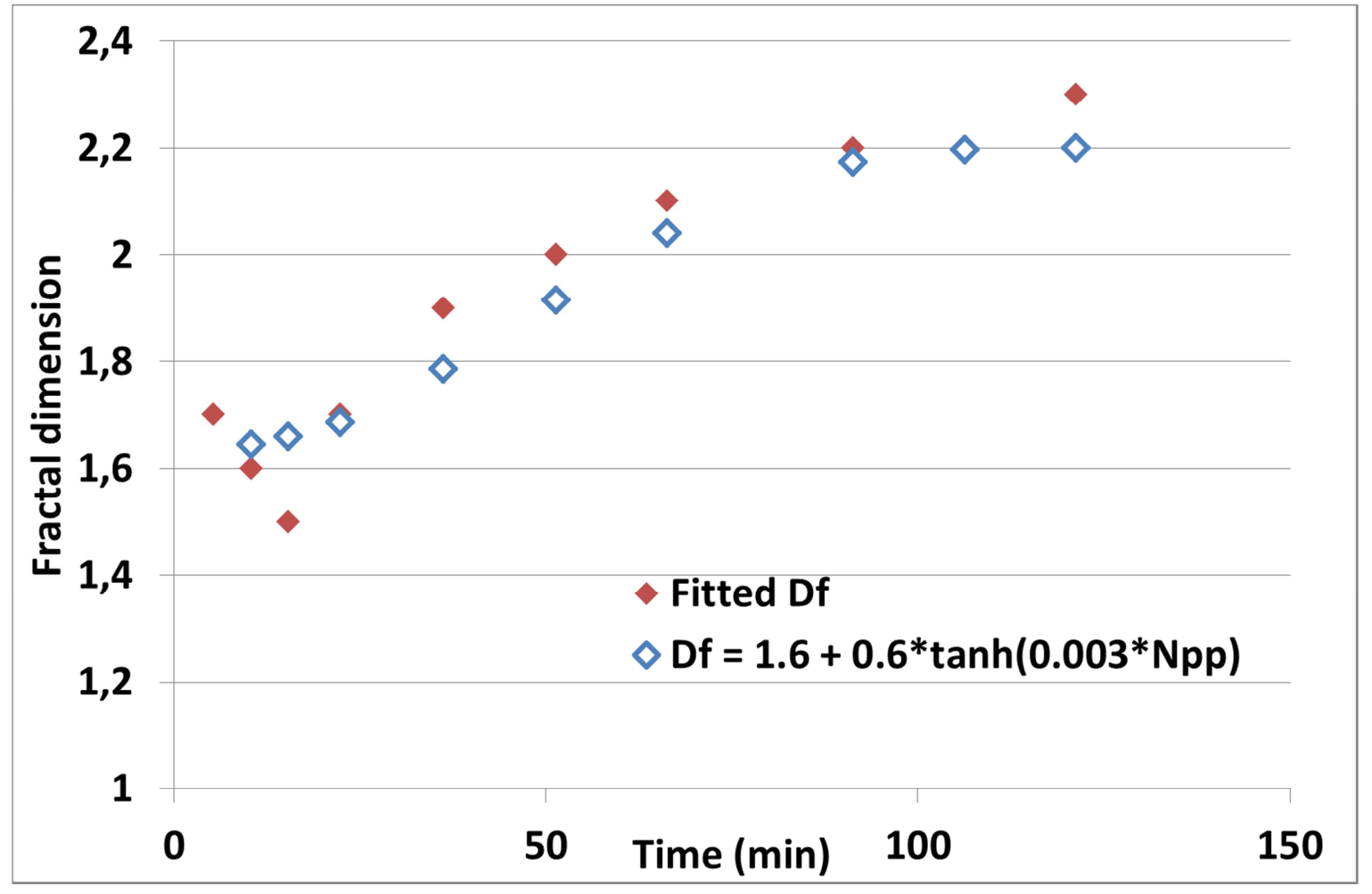

Figure 9: Comparison of the fitted Df with the values issued from $\operatorname{Df}(\mathrm{Npp})=1.6+0.6 * \tanh (0.003 * \mathrm{Npp})$ 


\begin{tabular}{|l|c|c|c|c|c|c|c|c|c|c|c|}
\hline Time (min) & 0 & 5 & 10 & 15 & 22 & 36 & 51 & 66 & 91 & 106 & 121 \\
\hline $\mathbf{N}_{\mathbf{p p}}(-)$ & 18 & 8 & 18 & 21 & 21 & 298 & 538 & 53 & 45 & 65 & 108 \\
\hline $\mathbf{r}_{\mathbf{p p}}(\mathbf{n m})$ & 3.7 & 5.4 & 4.7 & 4.8 & 5.3 & $\mathbf{3}$ & $\mathbf{3}$ & 7,4 & 9,5 & $\mathbf{1 0}$ & $\mathbf{1 0}$ \\
\hline $\mathbf{D}_{\mathbf{f}}(-)$ & 2.7 & $\mathbf{1}$ & 1.4 & 1.3 & 1.2 & 2.1 & 2.2 & 1.5 & 1.4 & 1.5 & 1.7 \\
\hline
\end{tabular}

Table 1: Aggregate morphological parameters extracted from the experimental turbidity spectra at different times of the experiment (Upper and lower limits are respectively $3 \mathrm{~nm}$ and $10 \mathrm{~nm}$ for rpp, 1 and 3 for Df) - pH 7.5 


\begin{tabular}{|l|l|c|c|c|c|c|c|c|c|c|c|c|}
\cline { 2 - 13 } \multicolumn{1}{c|}{} & $\begin{array}{l}\text { Time } \\
(\mathbf{m i n})\end{array}$ & 0 & 5 & 10 & 15 & 22 & 36 & 51 & 66 & 91 & 106 & 121 \\
\hline \multirow{2}{*}{$\mathbf{r}_{\mathrm{pp}}=\mathbf{4 . 2} \mathbf{~ n m}$} & $\mathbf{N}_{\mathrm{pp}}(-)$ & 12 & 18 & 25 & 33 & 48 & 107 & 194 & 312 & 627 & 1001 & 1606 \\
\cline { 2 - 14 } & $\mathbf{D}_{\mathbf{f}}(-)$ & 2.6 & 1.7 & 1.6 & 1.5 & 1.7 & 1.9 & 2.0 & 2.1 & 2.2 & 2.2 & 2.3 \\
\hline \multirow{2}{*}{$\mathbf{r}_{\mathbf{p p}}=\mathbf{5 . 0} \mathbf{~ n m}$} & $\mathbf{N}_{\mathbf{p p}}(-)$ & 7 & 10 & 14 & 17 & 26 & 62 & 113 & 181 & 363 & 581 & 932 \\
\cline { 2 - 13 } & $\mathbf{D}_{\mathbf{f}}(-)$ & 2.4 & 1.2 & 1.2 & 1.1 & 1.3 & 1.7 & 1.9 & 2.0 & 2.1 & 2.1 & 2.2 \\
\hline
\end{tabular}

Table 2: Aggregate morphological parameters extracted from the experimental turbidity spectra at different times of the experiment when rpp is fixed and Df is a fitting parameter (Upper and lower limits are 1 and 3 for Df. The calculations are performed successively for $\mathrm{rpp}=4.2 \mathrm{~nm}$ and $\mathrm{rpp}=5.0 \mathrm{~nm}$ ) $-\mathrm{pH}$ 7.5 


\section{Highlights}

- We monitored aggregation of silica particles thanks to turbidity and DLS analyses.

- We developed an optical model to quantatively interpret the turbidity spectra.

- Analysis of the data evidenced aggregate restructuring. 


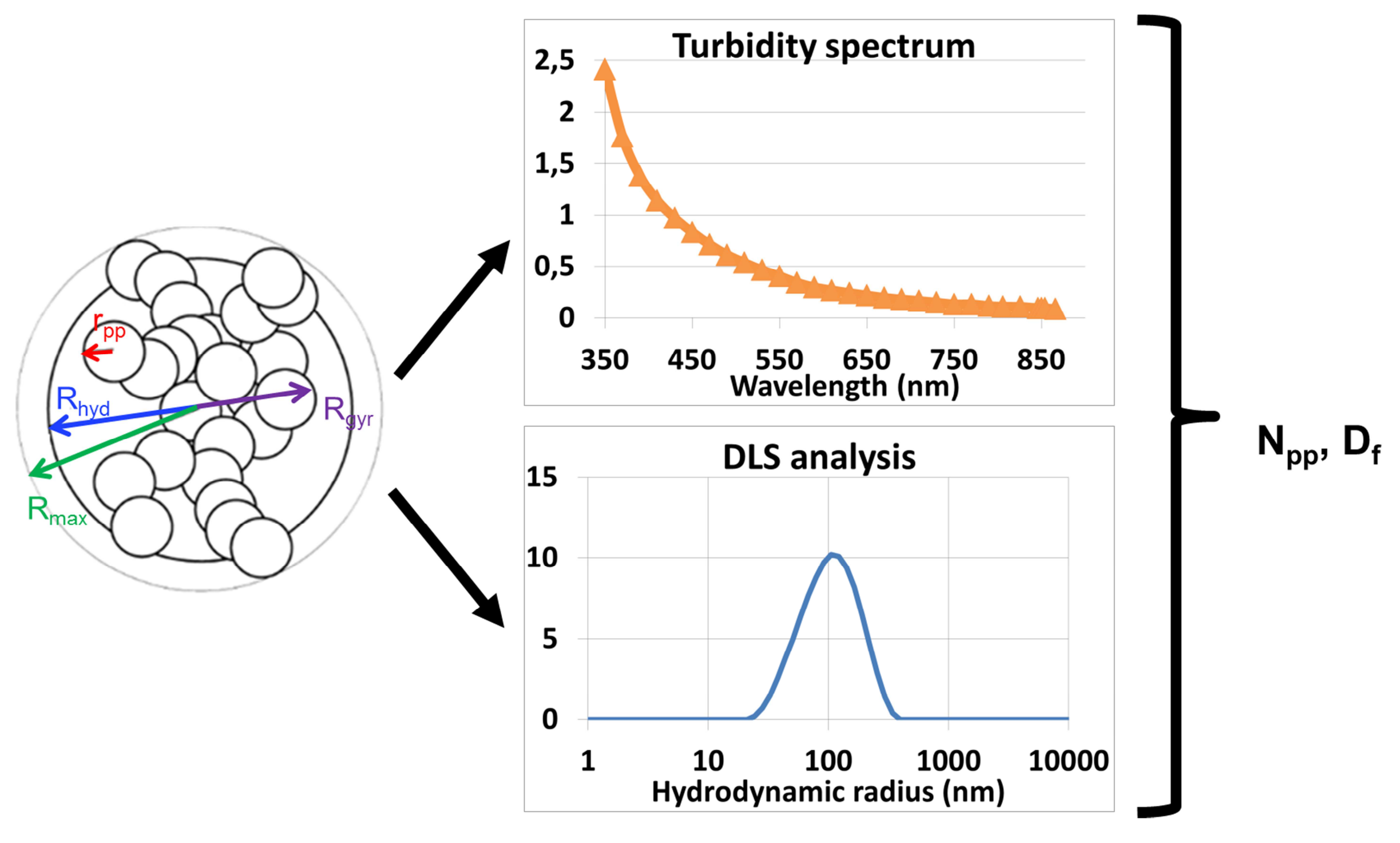

